\title{
The lag projective (anti-)synchronization of chaotic systems with bounded nonlinearity via an adaptive control scheme
}

\author{
Tianbo Wang ${ }^{1 *}$, Shouwei Zhao ${ }^{1}$, Wuneng Zhou ${ }^{2}$ and Weiqin Yu ${ }^{1}$
}

\section{"Correspondence:}

tb_wang@163.com

${ }^{1}$ College of Fundamental Studies,

Shanghai University of Engineering

Science, Shanghai, 201620,

P.R. China

Full list of author information is

available at the end of the article

\begin{abstract}
This paper investigates the lag projective synchronization and anti-synchronization problems for a general master-slave chaotic system with bounded nonlinearity. An adaptive controller is designed to guarantee that the slave system synchronizes with the master system by using Lyapunov stability theory and the idea of bang-bang control. Different from some existing master-slave models, the nonlinear terms in the considered chaotic system only need to satisfy bounded conditions. Furthermore, the structure of the master system does not need to match that of the slave system. Finally, three numerical examples are given to illustrate the main results.
\end{abstract}

\section{Introduction}

In the past few decades, the synchronization of master-slave chaotic systems has become a hot research topic in the nonlinear science field. One of the most important reasons is its potential applications in the real world such as the secure communications [1, 2], telecommunication [3-6], chemical reactions and biological systems [7, 8], etc. For a master-slave system, the synchronization means that the state of a slave system follows the state of a master system asymptotically by using the output of the master system to control the slave system. It is well known that the state trajectories of chaotic systems closely depend on the initial conditions, then it is difficult to drive a master-slave chaotic system with different initial conditions to achieve synchronization. In particular, for a master-slave chaotic system with different state equations, this problem becomes much more difficult.

To date, many papers on the study of the synchronization control of chaotic systems have appeared. For example, Su et al. investigated the synchronization of a discrete masterslave chaotic system with impulsive effect in [9], and Qi et al. investigated the $H_{\infty}$ synchronization of a discrete master-slave chaotic system with external disturbance in [10], respectively. In these two papers, the master system is required to match well the slave system; moreover, the nonlinear parts in the master-slave system must satisfy the Lipschitz condition. For continuous cases, there are also many related results. For instance, in [11] and [12], Yang et al. and $\mathrm{Xu}$ et al. studied the lag synchronization of the chaotic system with time delay. The anti-synchronization of chaotic systems is investigated in $[13,14]$ by using the adaptive control method. The projective synchronization of chaotic systems is studied by utilizing adaptive back-stepping control, state feedback control and impulsive control in [15-17], respectively. Similar results can be found in [18-28] and the references

\section{算 Springer}

(c) 2013 Wang et al.; licensee Springer. This is an Open Access article distributed under the terms of the Creative Commons Attribution License (http://creativecommons.org/licenses/by/2.0), which permits unrestricted use, distribution, and reproduction in any medium, provided the original work is properly cited. 
therein. In these papers, the chaotic synchronization problems mainly include synchronization, projective synchronization, anti-synchronization and lag synchronization. The used synchronization control methods mostly include the state feedback control, pinning control, adaptive control and impulsive control.

Although there have been a lot of literature works studying the synchronization problem of chaotic systems, we find the following deficiencies: (1) Most of them require the nonlinear parts in the chaotic systems to satisfy the Lipschitz condition. (2) Each paper usually only could solve a single synchronization problem, which leads to the lack of a unified method to solve all the synchronization problems for the same master-slave model. Motivated by these factors, we aim in this paper to find a kind of effective method to deal with all the synchronization problems for a general master-salve chaotic system. The contributions of this paper are as follows: (i) The lag projective synchronization which can include synchronization, projective synchronization, anti-synchronization and lag synchronization at the same time is investigated. (ii) The considered master-slave system model is different from the systems in the literature, in which the nonlinearities only need to satisfy a bounded condition. Moreover, the state equations of the master system and the slave system are non-identical. (iii) The presented results are very concise and it is easy to adjust the synchronization rate by the control gains.

The rest of this paper is organized as follows. In Section 2, the investigated masterslave chaotic system is presented and an adaptive synchronization controller is designed. In Section 3, three numerical examples are provided to illustrate the effectiveness of the obtained method. Conclusions are drawn in Section 4.

\section{Notations}

$R^{n}$ and $R^{n \times m}$ denote the $n$-dimensional Euclidean space and the set of $n \times m$ real matrices, respectively. $R_{+}$stands for the set of positive real numbers. For an $n$-dimensional vector $v=\left(v_{1}, v_{2}, \ldots, v_{n}\right)^{T} \in R^{n}$, whose norms are defined as $\|v\|_{1}=\sum_{i=1}^{n}\left|v_{i}\right|,\|v\|_{2}=\sqrt{\sum_{i=1}^{n} v_{i}^{2}}$ and $\|v\|_{\infty}=\max _{1 \leq i \leq n}\left\{\left|v_{i}\right|\right\}, \operatorname{sign}(v)=\left(\operatorname{sign}\left(v_{1}\right), \operatorname{sign}\left(v_{2}\right), \ldots, \operatorname{sign}\left(v_{n}\right)\right)^{T} \cdot A^{T}$ denotes the transpose of matrix $A$, the notation $X \geq Y$ (respectively, $X>Y$ ) means that $X-Y$ is a symmetric semi-definite matrix (respectively, positive definite matrix), where $X, Y$ are symmetric matrices. $I_{n}$ is the $n \times n$ identical matrix.

\section{Synchronization control scheme}

Consider a general master-slave chaotic dynamical system that is described as follows:

$$
\left\{\begin{array}{l}
\text { Master: } \dot{x}(t)=A x(t)+f(x(t), t), \\
\text { Slave: } \dot{y}(t)=B y(t)+g(y(t), t)+u(t),
\end{array}\right.
$$

where $x(t)=\left(x_{1}(t), x_{2}(t), \ldots, x_{n}(t)\right)^{T} \in R^{n}$ and $y(t)=\left(y_{1}(t), y_{2}(t), \ldots, y_{n}(t)\right)^{T} \in R^{n}$ are the state vectors of the master system and the slave system, respectively. $A \in R^{n \times n}$ and $B \in$ $R^{n \times n}$ are two known real matrices, $f(\cdot): R^{n} \times R_{+} \rightarrow R^{n}$ and $g(\cdot): R^{n} \times R_{+} \rightarrow R^{n}$ are two bounded nonlinear vector functions. $u(t) \in R^{n}$ is the control input to be determined.

For given scalars $\tau \geq 0$ and $\mu \neq 0$, define the error state as $e(t)=y(t)+\mu x(t-\tau)$, then one can obtain the following error system:

$$
\dot{e}(t)=A e(t)+g(y(t), t)+\mu f(x(t-\tau), t-\tau)+(B-A) y(t)+u(t) .
$$


Definition 1 For given constants $\tau$ and $\mu$, master-salve chaotic system (1) is said to be lag projective synchronization if

$$
\lim _{t \rightarrow+\infty}\|e(t)\|_{2}=0
$$

for any initial conditions.

Remark 1 The aim of this paper is to design a suitable controller $u(t)$ such that the controlled slave system synchronizes with the master system. Thus, by Definition 1 , one only needs to design a controller such that error system (2) is stable.

Remark 2 Especially, master-salve chaotic system (1) is said to be lag anti-synchronization if $\mu=1$; master-salve chaotic system (1) is said to be lag synchronization if $\mu=-1$. When time delay $\tau=0$, master-salve chaotic system (1) is said to be anti-synchronization if $\mu=1$; master-salve chaotic system (1) is said to be synchronization if $\mu=-1$; master-salve chaotic system (1) is said to be projective synchronization if $\mu \neq \pm 1$. Then Definition 1 unites several kinds of synchronization definitions together and is very general.

In order to study the lag projective synchronization of master-slave chaotic system (1), the following assumption is firstly given.

Assumption 1 Assume that the nonlinear functions in master-slave system (1) are bounded, i.e., there exist two constants $M_{f} \geq 0$ and $M_{g} \geq 0$ such that

$$
\|f(x(t), t)\|_{\infty} \leq M_{f}, \quad\|g(y(t), t)\|_{\infty} \leq M_{g}
$$

hold for any $x(t), y(t) \in R^{n}$ and $t>0$.

Remark 3 There exist a lot of papers that study the synchronization problems of masterslave chaotic systems. Different from them, the nonlinear functions $f(x(t), t)$ and $g(y(t), t)$ in system (1) need not satisfy the Lipschitz condition. In fact, the bounded functions could satisfy the Lipschitz condition. For example, $f(z)=\sin \frac{1}{z}$ is bounded but does not satisfy the Lipschitz condition in the neighborhood of zero. Conversely, the functions satisfying the Lipschitz condition may not be bounded. For instance, $f(z)=z$ satisfies the Lipschitz condition but it is not bounded. Therefore, the Lipschitz condition and bounded condition are different. To the best of our knowledge, the results on this topic are few.

In this paper, the following controller and updated laws are designed

$$
\left\{\begin{array}{l}
u(t)=-(B-A) y(t)-L_{1}(t) e(t)-L_{2}(t) \cdot \operatorname{sign}(P e(t)), \\
\dot{L}_{1}(t)=\alpha e^{T}(t) \operatorname{Pe}(t), \\
\dot{L}_{2}(t)=\beta\|\operatorname{Pe}(t)\|_{1}
\end{array}\right.
$$

to promote master-slave system (1) achieving synchronization, where $\alpha$ and $\beta$ are any given positive constants, $P \in R^{n \times n}$ is a positive definite symmetric matrix to be determined.

In what follows, the main result is obtained. 
Theorem 1 Suppose master-slave chaotic system (1) satisfies Assumption 1. If there exist scalars $L_{1}^{*}>0, L_{2}^{*}>0$ and a positive definite symmetric matrix $P \in R^{n \times n}$ such that

$$
P A+A^{T} P-2 L_{1}^{*} P<0
$$

and

$$
M_{f}|\mu|+M_{g}<L_{2}^{*}
$$

hold, then master-slave chaotic system (1) synchronizes well under the action of adaptive controller (4), where $\alpha$ and $\beta$ are any given positive constants.

Proof Choose the following Lyapunov function:

$$
V(e(t))=e^{T}(t) P e(t)+\frac{1}{\alpha}\left(L_{1}(t)-L_{1}^{*}\right)^{2}+\frac{1}{\beta}\left(L_{2}(t)-L_{2}^{*}\right)^{2},
$$

where $L_{1}^{*}$ and $L_{2}^{*}$ are sufficiently large positive constants to be determined in the sequel. The derivative of $V(e(t))$ along the state trajectories of system (2) is

$$
\begin{aligned}
\dot{V}(e(t))= & \dot{e}^{T}(t) P e(t)+e^{T}(t) P \dot{e}(t)+2 \alpha^{-1}\left(L_{1}(t)-L_{1}^{*}\right) \dot{L}_{1}(t)+2 \beta^{-1}\left(L_{2}(t)-L_{2}^{*}\right) \dot{L}_{2}(t) \\
= & {[A e(t)+g(y(t), t)+\mu f(x(t-\tau), t-\tau)} \\
& +(B-A) y(t)+u(t)]^{T} P e(t)+e^{T}(t) P[A e(t)+g(y(t), t) \\
& +\mu f(x(t-\tau), t-\tau)+(B-A) y(t)+u(t)] \\
& +2\left(L_{1}(t)-L_{1}^{*}\right) e^{T}(t) P e(t)+2\left(L_{2}(t)-L_{2}^{*}\right)\|P e(t)\|_{1} \\
= & e^{T}(t)\left(P A+A^{T} P\right) e(t)+g^{T}(y(t), t) P e(t)+e^{T}(t) \operatorname{Pg}(y(t), t) \\
& +\mu\left[f^{T}(x(t-\tau), t-\tau) \operatorname{Pe}(t)+e^{T}(t) P f(x(t-\tau), t-\tau)\right] \\
& -2 e^{T}(t) P\left[L_{1}(t) e(t)+L_{2}(t) \cdot \operatorname{sign}(\operatorname{Pe}(t))\right] \\
& +2\left(L_{1}(t)-L_{1}^{*}\right) e^{T}(t) P e(t)+2\left(L_{2}(t)-L_{2}^{*}\right)\|P e(t)\|_{1} \\
= & e^{T}(t)\left(P A+A^{T} P\right) e(t)+g^{T}(y(t), t) P e(t)+e^{T}(t) P g(y(t), t) \\
& +\mu\left[f^{T}(x(t-\tau), t-\tau) P e(t)+e^{T}(t) P f(x(t-\tau), t-\tau)\right] \\
& -2 L_{1}^{* \prime} e^{T}(t) P e(t)-2 L_{2}^{*}\|P e(t)\|_{1} .
\end{aligned}
$$

From Assumption 1, one gets

$$
g^{T}(y(t), t) \operatorname{Pe}(t)+e^{T}(t) \operatorname{Pg}(y(t), t) \leq 2\|g(y(t), t)\|_{\infty} \cdot\|P e(t)\|_{1} \leq 2 M_{g}\|P e(t)\|_{1}
$$

and

$$
\begin{aligned}
\mu & {\left[f^{T}(x(t-\tau), t-\tau) \operatorname{Pe}(t)+e^{T}(t) P f(x(t-\tau), t-\tau)\right] } \\
& \leq 2|\mu| \cdot\|f(x(t-\tau), t-\tau)\|_{\infty} \cdot\|\operatorname{Pe}(t)\|_{1} \\
& \leq 2 M_{f}|\mu| \cdot\|P e(t)\|_{1} .
\end{aligned}
$$


Inequalities (9) and (10) yield that

$$
\dot{V}(e(t)) \leq e^{T}(t)\left(P A+A^{T} P-2 L_{1}^{*} P\right) e(t)+2\left(M_{f}|\mu|+M_{g}-L_{2}^{*}\right)\|P e(t)\|_{1} .
$$

From inequalities (5) and (6), we have

$$
\dot{V}(e(t))<0 \text {. }
$$

By the Lasalle invariance principle of differential equation, it is known that all the state trajectories of system (1) converge to the set $S=\{e(t) \mid e(t)=0\}$, which implies that $\lim _{t \rightarrow+\infty}\|e(t)\|_{2}=0$ and the slave system synchronizes with the master system. This completes the proof.

Remark 4 Adaptive controller (4) is divided into three parts. The first and second parts can be thought of as the state feedback control, and the last part can be thought of as a suitable complement for the bounded nonlinearities in the chaotic system by using the idea of the bang-bang control.

Remark 5 It is easy to see that adaptive controller (4) can always drive the slave system to synchronize with the master system since inequalities (5) and (6) always have feasible solutions. Different from the methods in $[28,29]$, our method does not need to do some complex computation. Furthermore, the synchronization rate can be altered through adjusting the control gains $\alpha$ and $\beta$.

Remark 6 From Theorem 1, it is also easy to design the synchronization controller, antisynchronization controller, lag synchronization controller and projective synchronization controller for chaotic system (1).

\section{Numerical simulations}

In this section, three examples are given to show the effectiveness of the proposed method.

Example 1 Consider the known second-order non-autonomous chaotic system [30]

$$
\ddot{y}(t)+\delta[d \cos 2 y(t)-d+1] \dot{y}(t)+\left(c^{2}-\cos y(t)\right) \sin y(t)=\delta \Gamma \cos w t,
$$

where $c, d, \delta, w, \Gamma$ are positive constants.

Let $y(t)=x_{1}(t)$ and $\dot{y}(t)=x_{2}(t)$, then system (12) can be transformed to

$$
\left\{\begin{array}{l}
\dot{x}_{1}(t)=x_{2}(t), \\
\dot{x}_{2}(t)=-\delta\left[d \cos 2 x_{1}(t)-d+1\right] x_{2}(t)-\left(c^{2}-\cos x_{1}(t)\right) \sin x_{1}(t)+\delta \Gamma \cos w t
\end{array}\right.
$$

or

$$
\dot{x}(t)=A x(t)+f(x(t), t),
$$

where $x(t)=\left(x_{1}(t), x_{2}(t)\right)^{T}, A=\left[\begin{array}{cc}0 & 1 \\ 0 & \delta(d-1)\end{array}\right], f(x(t), t)=\left(0,-\delta d \cos 2 x_{1}(t) \cdot x_{2}(t)-\left(c^{2}-\right.\right.$ $\left.\left.\cos x_{1}(t)\right) \sin x_{1}(t)+\delta \Gamma \cos w t\right)^{T}$. When choosing $c=0.07338, d=0.8, \delta=0.1$ and $w=\Gamma=1$, 


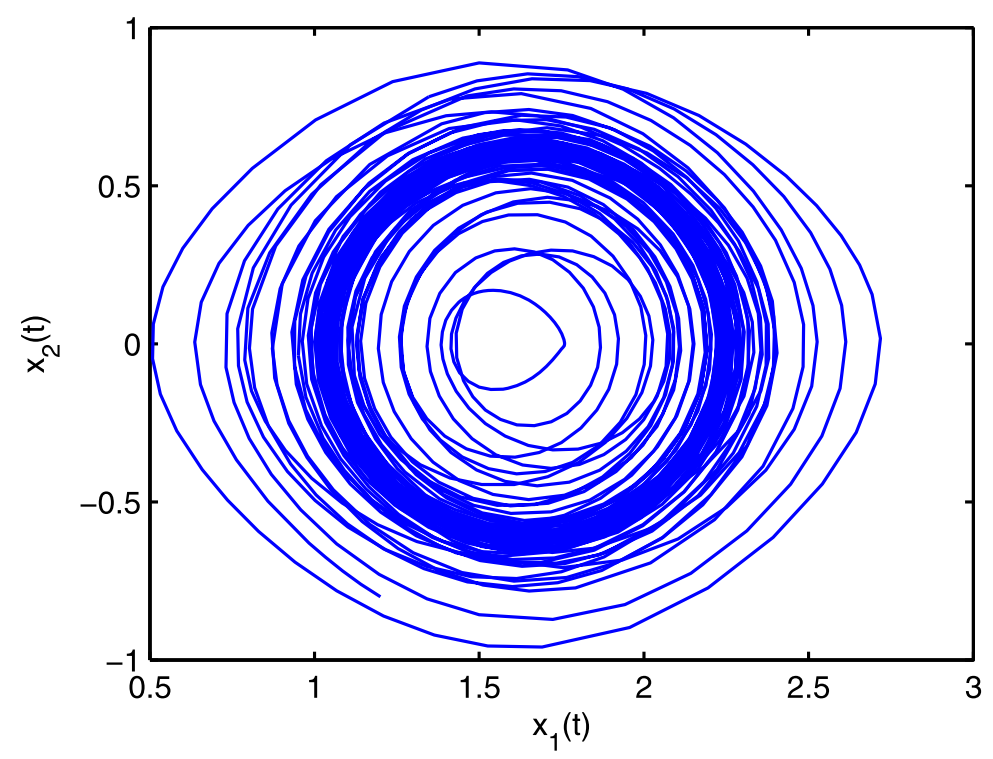

Figure 1 Phase diagram of system (13) with initial condition $x(0)=(1.2,-0.8)^{T}$.

the phase diagram of system (13) is shown in Figure 1. This figure shows that the state trajectories are bounded for $t>0$, then $f(x(t), t)$ is bounded for all the $x(t)$ and Assumption 1 is satisfied. Taking two non-autonomous systems defined by (12) to constitute a masterslave system, setting $\mu=-1$ and $\tau=2$, and computing by the LMI's Toolbox in the Matlab, the following feasible solutions

$$
P=\operatorname{diag}\{1,1\}, \quad L_{1}^{*}=0.1, \quad L_{2}^{*}=0.5
$$

are obtained. Under the action of adaptive controller (4), taking the initial conditions as $x(0)=(1.2,-0.8)^{T}$ and $y(0)=(-12,8)^{T}, \alpha=\beta=0.1$, we show the state trajectories of error system (2) and updated laws in adaptive controller (4) in Figures 2-4, respectively. These figures show that the master-slave system is lag synchronization.

Remark 7 In [30], Wu et al. investigated a synchronization problem of the master-slave system composed of system (12). Using their methods, one needs to do some complex computation and discussion. Hence, our method is more concise than theirs.

Example 2 Consider the following Chua's circuit [29]:

$$
\left\{\begin{array}{l}
\dot{x}_{1}(t)=a\left[x_{2}(t)-m_{1} x_{1}(t)\right]-a h\left(x_{1}(t)\right), \\
\dot{x}_{2}(t)=x_{1}(t)-x_{2}(t)+x_{3}(t), \\
\dot{x}_{3}(t)=-b x_{2}(t)
\end{array}\right.
$$

where $h\left(x_{1}(t)\right)=\frac{1}{2}\left(m_{0}-m_{1}\right)\left(\left|x_{1}(t)+c\right|-\left|x_{1}(t)-c\right|\right), a=9, b=14.28, c=1, m_{0}=-1 / 7$ and $m_{1}=2 / 7$. System (14) can be represented by

$$
\dot{x}(t)=A x(t)+f(x(t), t),
$$



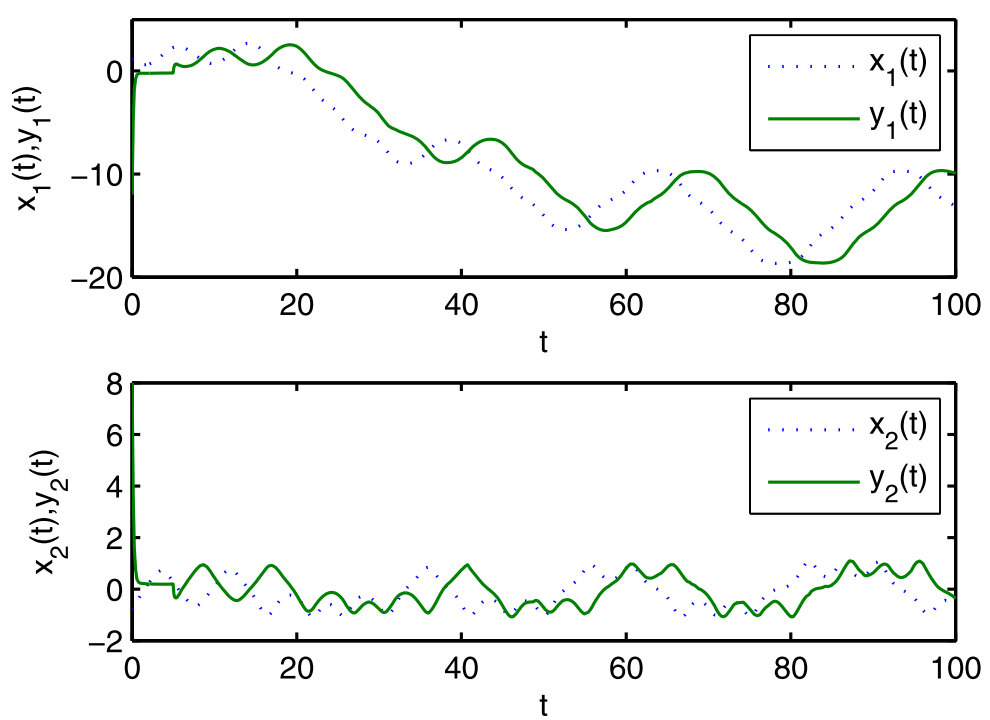

Figure 2 State trajectories of the master-slave system with initial conditions $x(0)=(1.2,-0.8)^{T}$ and $y(0)=(-12,8)^{T}$

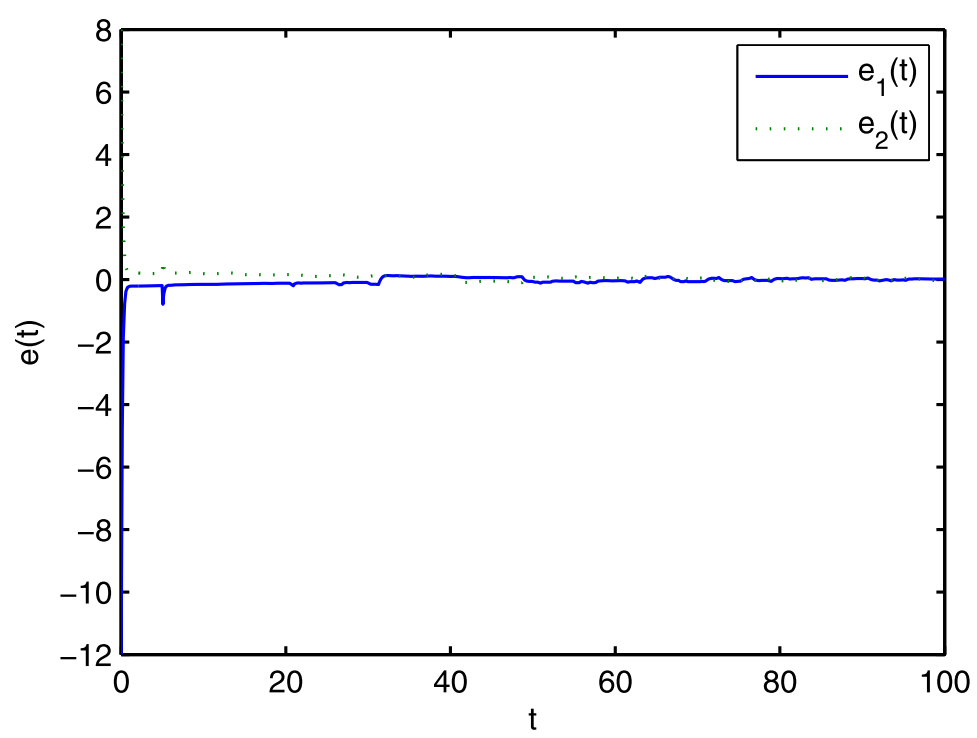

Figure 3 Synchronization error trajectories.

where $A=\left[\begin{array}{rrr}-a m_{1} & a & 0 \\ 1 & -1 & 1 \\ 0 & -b & 0\end{array}\right], f(x(t), t)=\left(-a h\left(x_{1}(t)\right), 0,0\right)^{T}$. Taking this system as the master system, the corresponding slave system is

$$
\dot{y}(t)=A y(t)+g(y(t), t)
$$

where $g(y(t), t)=\left(0, h\left(y_{2}(t)\right), 0\right)^{T}$. Since

$$
\left|x_{1}(t)+c\right|-\left|x_{1}(t)-c\right| \leq\left|\left(x_{1}(t)+c\right)-\left(x_{1}(t)-c\right)\right|=2 c
$$




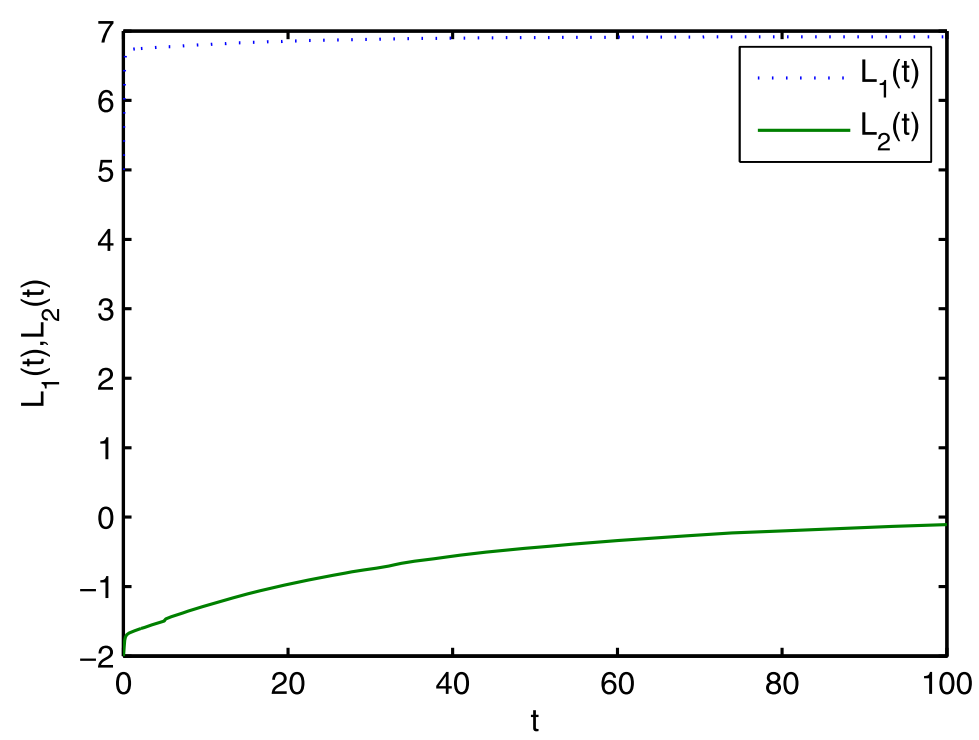

Figure 4 State trajectories of updated laws.
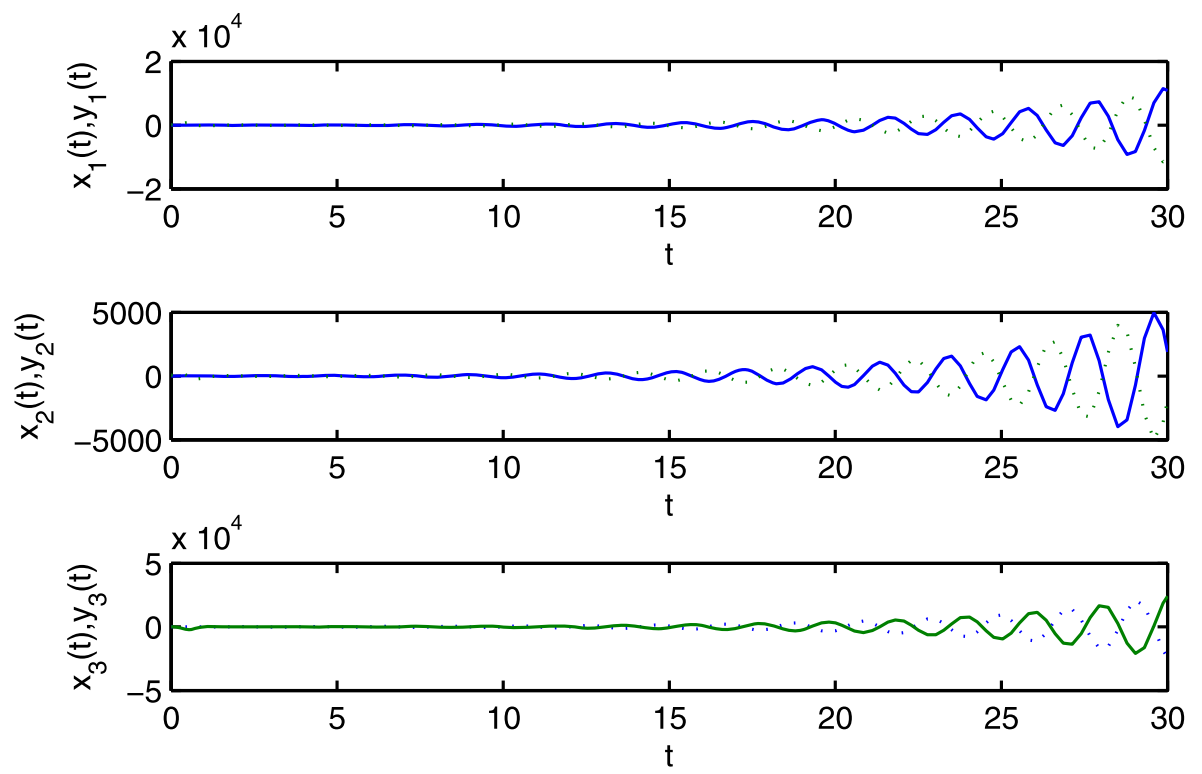

Figure 5 State trajectories of the master-slave system with initial conditions $x(0)=(20,-20,50)^{T}$ and $y(0)=(40,70,-50)^{T}$.

then $\|f(x(t), t)\|_{\infty}=\|g(y(t), t)\|_{\infty} \leq a c\left|m_{0}-m_{1}\right|$, which implies that Assumption 1 holds. Considering the anti-synchronization of the master-slave system composed of system (14), choosing $\mu=1, \tau=0$, and computing by the LMI's Toolbox in the Matlab, one can obtain the feasible solutions of inequalities (5) and (6) as follows:

$$
P=\operatorname{diag}\{1,1,1\}, \quad L_{1}^{*}=7.5, \quad L_{2}^{*}=8 .
$$




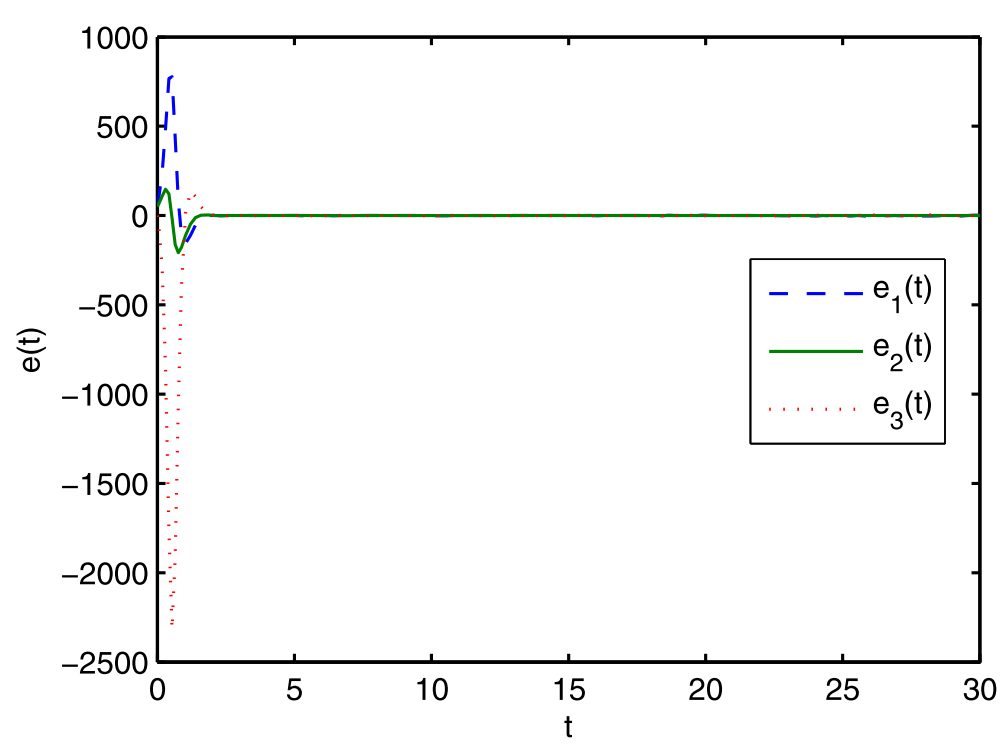

Figure 6 Anti-synchronization error trajectories.

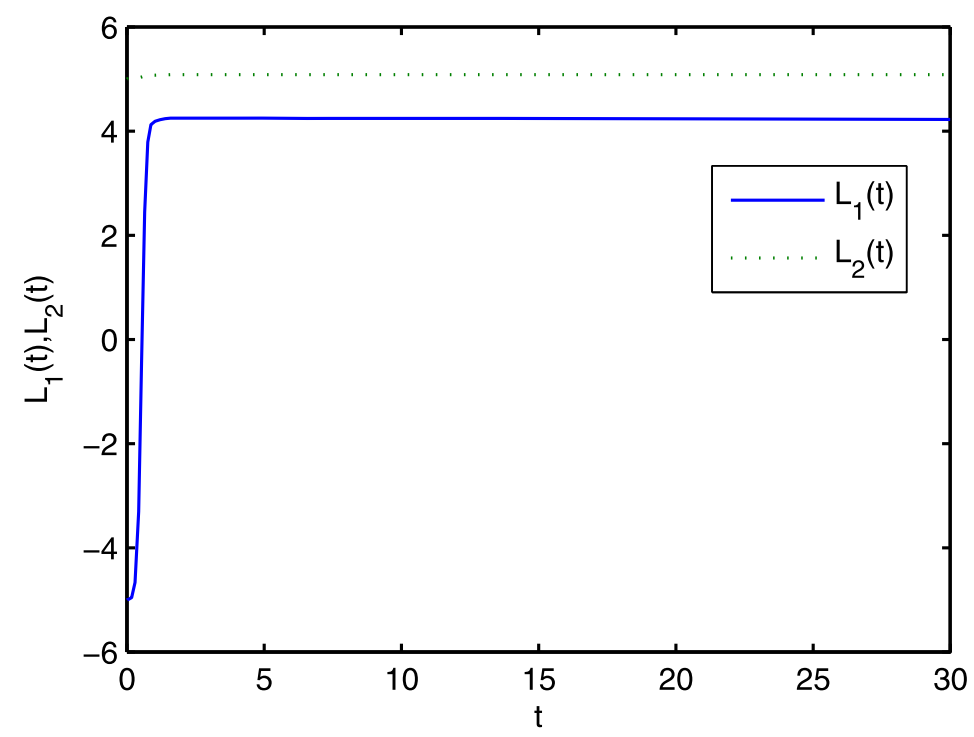

Figure 7 State trajectories of updated laws.

Taking the initial conditions as $x(0)=(20,-20,50)^{T}$ and $y(0)=(40,70,-50)^{T}, \alpha=\beta=0.5$, under the action of adaptive controller (4), we show the illustration results in Figures 5-7, respectively. These figures show that the master-slave system is anti-synchronization.

Example 3 Consider the following dynamical system:

$$
\dot{x}(t)=A x(t)+f(x(t), t),
$$



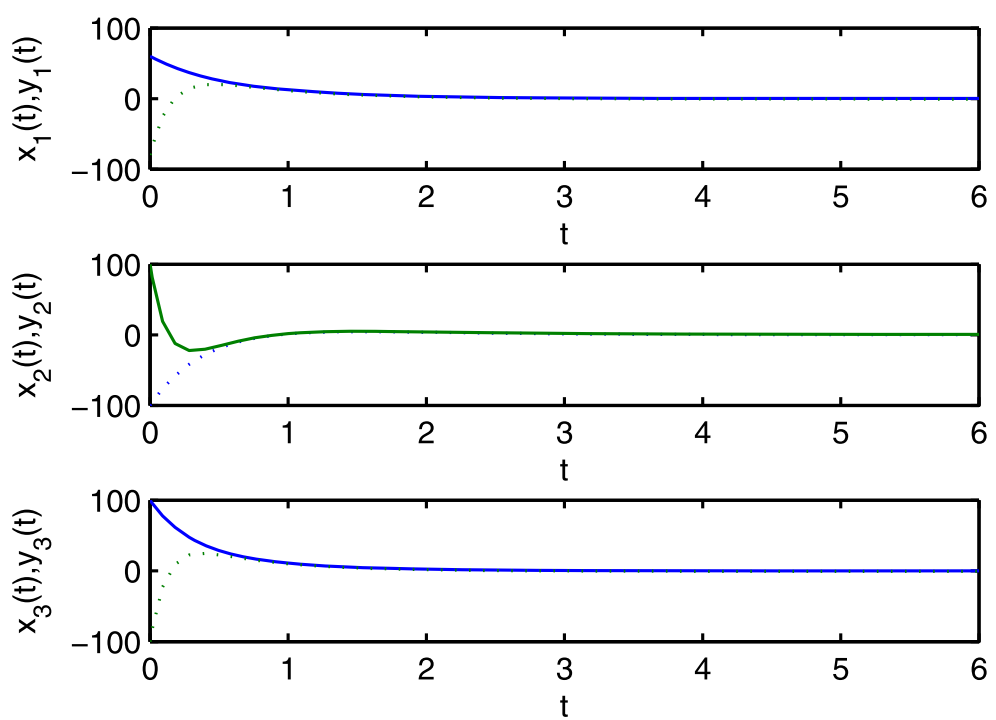

Figure 8 State trajectories of the master-slave system with initial conditions $x(0)=(60,-100,100)^{T}$ and $y(0)=(-80,100,-100)^{T}$.

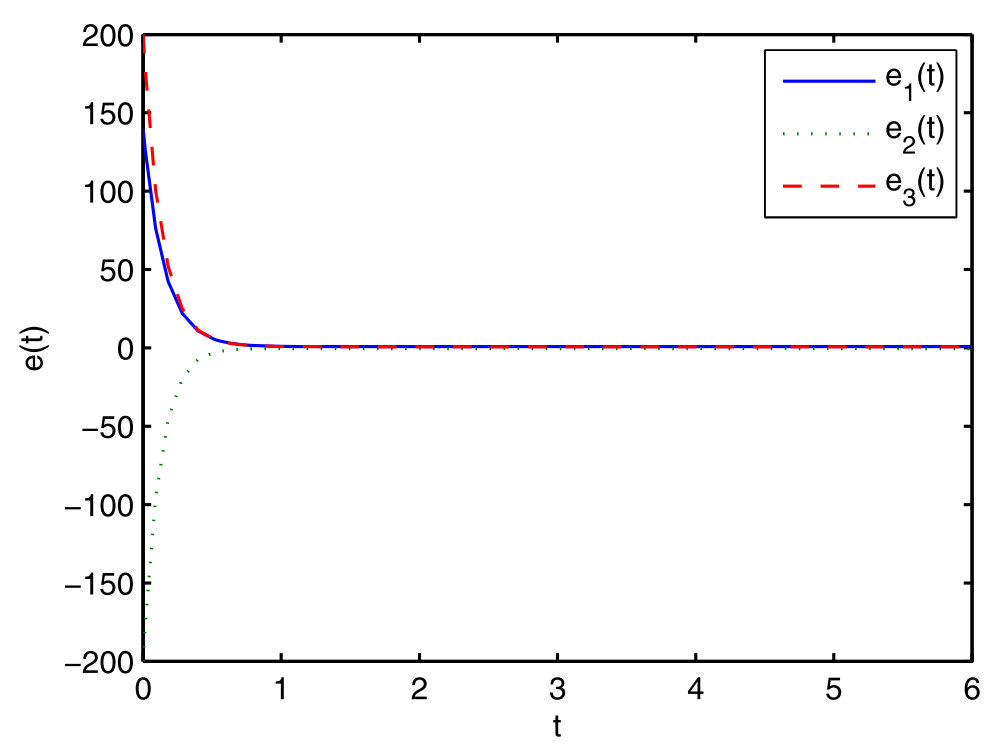

Figure 9 Synchronization error trajectories.

where $A=\left[\begin{array}{ccc}-1 & 0 & -0.5 \\ 1.5 & -2 & 0 \\ 2 & 0 & -4\end{array}\right], f(x(t), t)=\left(\sin \frac{1}{\left|x_{1}(t)\right|+\varepsilon}, \sin \frac{1}{\left|x_{2}(t)\right|+\varepsilon}, \sin \frac{1}{\left|x_{3}(t)\right|+\varepsilon}\right)^{T}, \varepsilon=0.00001$. Taking system (15) as the master system, and $\dot{y}(t)=A y(t)-g(y(t), t)$ as the slave system, where $g(y(t), t)=\left(\cos \frac{1}{\left|y_{1}(t)\right|+\varepsilon}, \cos \frac{1}{\left|y_{2}(t)\right|+\varepsilon}, \cos \frac{1}{\left|y_{3}(t)\right|+\varepsilon}\right)^{T}$, it is easy to see $\|f(x(t), t)\|_{\infty}=$ $\|g(y(t), t)\|_{\infty} \leq 1$ for any $x(t), y(t) \in R^{3}$, and $f(x(t), t)$ and $g(y(t), t)$ do not satisfy the Lipschitz condition. Considering the synchronization of this master-slave system and choosing $\mu=-1, \tau=0$, after computing by the LMI's Toolbox in the Matlab, one can get the feasible solutions of inequalities (5) and (6) as follows:

$$
P=\operatorname{diag}\{1,1,1\}, \quad L_{1}^{*}=4, \quad L_{2}^{*}=1.1
$$




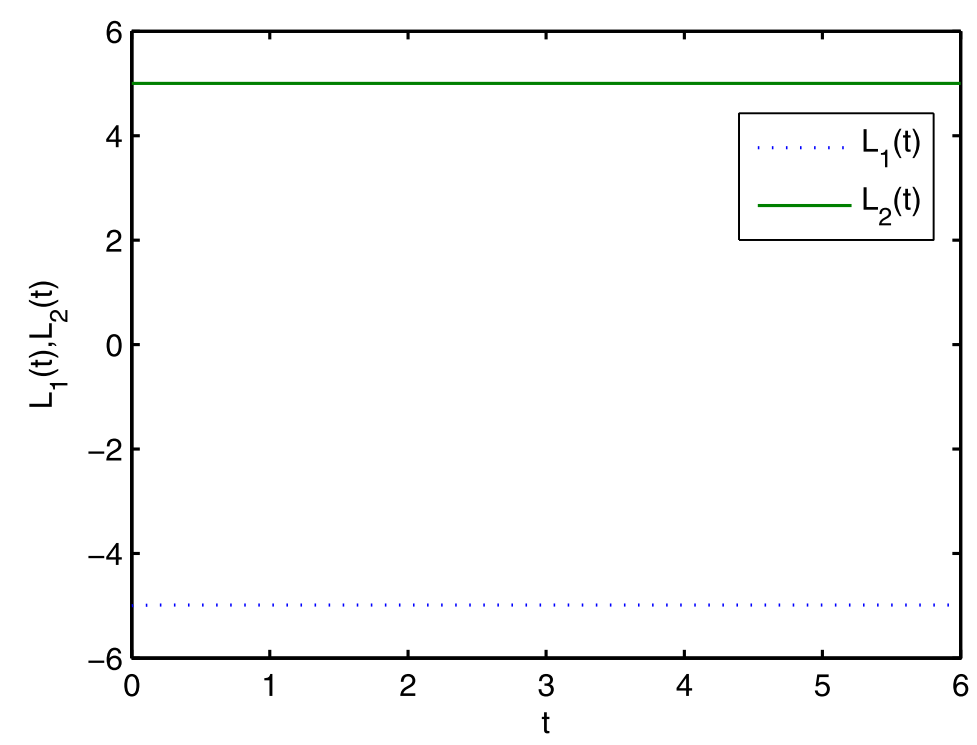

Figure 10 State trajectories of updated laws.

Taking the initial conditions as $x(0)=(60,-100,100)^{T}$ and $y(0)=(-80,100,-100)^{T}, \alpha=$ $\beta=0.2$, under the action of adaptive controller (4), we show the illustration results in Figures 8-10. These figures show that the master-slave system is synchronization. It should be noticed that the nonlinear function in system (15) is bounded but does not satisfy the Lipschitz condition.

\section{Conclusions}

In this paper, the lag projective (anti-)synchronization problems for a kind of master-slave chaotic systems by using the adaptive control method have been investigated. Based on the Lasalle invariance principle of differential equation and the idea of the bang-bang control, an adaptive controller with simple updated laws has been proposed. Three numerical examples have shown that the obtained method is effective.

Competing interests

The authors declare that they have no competing interests.

\section{Authors' contributions}

TW and SZ drafted the manuscript, WZ and WY performed the numerical illustration. All authors read and approved the final manuscript.

\section{Author details}

${ }^{1}$ College of Fundamental Studies, Shanghai University of Engineering Science, Shanghai, 201620, P.R. China. ${ }^{2}$ College of Information Sciences and Technology, Donghua University, Shanghai, 200051, P.R. China.

\section{Acknowledgements}

This work is supported by the Connotative Construction Project of Shanghai University of Engineering Science (nhky-2012-13).

Received: 16 March 2013 Accepted: 5 July 2013 Published: 24 July 2013

\section{References}

1. Sundar, S, Minai, A: Synchronization of randomly multiplexed chaotic systems with application to communication. Phys. Rev. Lett. 85, 5456-5459 (2000)

2. Bowong, S, Kakmeni, FM, Fotsin, H: A new adaptive observer-based synchronization scheme for private communication. Phys. Lett. A 355, 193-201 (2006) 
3. Li, ZJ, Cao, XQ, Ding, N: Adaptive fuzzy control for synchronization of nonlinear teleoperators with stochastic time-varying communication delays. IEEE Trans. Fuzzy Syst. 19, 745-757 (2011)

4. Song, QK, Cao, JD: Synchronization of nonidentical chaotic neural networks with leakage delay and mixed time-varying delays. Adv. Differ. Equ. 2011, 16 (2011)

5. Dimassi, H, Loria, A: Adaptive unknown-input observers-based synchronization of chaotic systems for telecommunication. IEEE Trans. Circuits Syst. I, Regul. Pap. 58, 800-812 (2011)

6. Hua, CC, Liu, XP: Delay-dependent stability criteria of teleoperation systems with asymmetric time-varying delays. IEEE Trans. Robot. 26, 925-932 (2010)

7. Pikovsky, A, Rosenblum, M, Kurths, J: Synchronization: A Universal Concept in Nonlinear Sciences. Cambridge University Press, Cambridge (2001)

8. Pecora, LM, Carroll, TL: Synchronization in chaotic systems. Phys. Rev. Lett. 64, 821-824 (1990)

9. Su, H, Ding, XH: Synchronization in time-discrete delayed chaotic systems. Neurocomputing 73, $478-483$ (2009)

10. Qi, DL, Liu, MQ, Qiu, MK, Zhang, SL: Exponential $H_{\infty}$ synchronization of general discrete-time chaotic neural networks with or without time delays. IEEE Trans. Neural Netw. 21, 1358-1364 (2010)

11. Yang, XS, Zhu, QX, Huang, CX: Lag stochastic synchronization of chaotic mixed time-delayed neural networks with uncertain parameters or perturbations. Neurocomputing 74, 1617-1625 (2011)

12. $\mathrm{Xu}, \mathrm{YH}, \mathrm{Zhou}, \mathrm{WN}$, Fang, JA, Sun, W: Adaptive lag synchronization and parameters adaptive lag identification of chaotic systems. Phys. Lett. A 374, 3441-3446 (2010)

13. Li, XF, Leung, CS, Han, XP, Liu, XJ, Chu, YD: Complete (anti-)synchronization of chaotic systems with fully uncertain parameters by adaptive control. Nonlinear Dyn. 63, 263-275 (2011)

14. Al-sawalha, MM, Noorani, MSM: Adaptive reduced-order anti-synchronization of chaotic systems with fully unknown parameters. Commun. Nonlinear Sci. Numer. Simul. 15, 3022-3034 (2010)

15. Farivar, F, Shoorehdeli, MA, Nekoui, MA, Teshnehlab, M: Chaos control and modified projective synchronization of unknown heavy symmetric chaotic gyroscope systems via Gaussian radial basis adaptive backstepping control. Nonlinear Dyn. 67, 1913-1941 (2012)

16. Du, HY, Zeng, QS, Wang, CH, Ling, MX: Function projective synchronization in coupled chaotic systems. Nonlinear Anal., Real World Appl. 11, 705-712 (2010)

17. Cao, JD, Daniel, WCH, Yang, YQ: Projective synchronization of a class of delayed chaotic systems via impulsive control. Phys. Lett. A 373, 3128-3133 (2009)

18. Li, XD, Ding, CM, Zhu, QX: Synchronization of stochastic perturbed chaotic neural networks with mixed delays. J. Franklin Inst. 347, 1266-1280 (2010)

19. Yassen, MT: Adaptive chaos control and synchronization for uncertain new chaotic dynamical system. Phys. Lett. A 350, 36-43 (2006)

20. Chen, SH, Yang, Q, Wang, CP: Impulsive control and synchronization of unified chaotic system. Chaos Solitons Fractals $20,751-758(2004)$

21. $\mathrm{Wu}, \mathrm{RC}, \mathrm{Liu}, \mathrm{JL}$ : Isochronal function projective synchronization between chaotic and time-delayed chaotic systems. Adv. Differ. Equ. 2012, 37 (2012)

22. Lee, SM, Ji, DH, Park, JH, Won, SC: $H_{\infty}$ Synchronization of chaotic systems via dynamic feedback approach. Phys. Lett. A 372, 4905-4912 (2008)

23. Zhang, $\mathrm{D}, \mathrm{Xu}$, J: Projective synchronization of different chaotic time-delayed neural networks based on integral sliding mode controller. Appl. Math. Comput. 217, 164-174 (2010)

24. Choi, YP, Ha, SY, Yun, SB: Complete synchronization of Kuramoto oscillators with finite inertia. Physica D 240, $32-44$ (2011)

25. Xia, YH, Yang, ZJ, Han, MA: Lag synchronization of unknown chaotic delayed Yang-Yang-Type fuzzy neural networks with noise perturbation based on adaptive control and parameter identification. IEEE Trans. Neural Netw. 20, 1165-1180 (2009)

26. Feng, GZ, Cao, JD: Master-slave synchronization of chaotic systems with a modified impulsive controller. Adv. Differ. Equ. 2013, 24 (2013)

27. Pan, L, Zhou, WN, Fang, JA, Li, DQ: Synchronization and anti-synchronization of new uncertain fractional-order modified unified chaotic systems via novel active pinning control. Commun. Nonlinear Sci. Numer. Simul. 15, 3754-3762 (2010)

28. Gan, QT, Xu, R, Kang, XB: Synchronization of chaotic neural networks with mixed time delays. Commun. Nonlinear Sci. Numer. Simul. 16, 966-974 (2011)

29. Huang, H, Feng, G, Cao, JD: Exponential synchronization of chaotic Lur'e systems with delayed feedback control. Nonlinear Dyn. 57, 441-453 (2009)

30. Wu, XF, Gui, ZF, Lin, Q, Cai, JP: A new Lyapunov approach for global synchronization of non-autonomous chaotic systems. Nonlinear Dyn. 59, 427-432 (2010)

doi:10.1186/1687-1847-2013-225

Cite this article as: Wang et al.: The lag projective (anti-)synchronization of chaotic systems with bounded nonlinearity via an adaptive control scheme. Advances in Difference Equations 2013 2013:225. 